\title{
Viaje al corazón de Taulabé
}

César Mauricio Urbizo Vivas*, Patrick Durán Leiva**${ }^{* *}$
Giovanni Peraldo Huertas ${ }^{* *}$, Ronald Ramírez Salazar**

\section{RESUMEN}

El presente trabajo es el resultado de una excursión en la que participaron académicos de la Universidad Nacional Autónoma de Honduras (UNAH), de la Universidad de Costa Rica (UCR) y del Grupo Espeleológico Anthros.

Se realizó una visita exploratoria al interior de las Cuevas de Taulabé para obtener evidencia científica que explique el origen y composición de dichas cavernas, con el fin de fortalecer los vínculos académicos interuniversitarios y con grupos especializados en espeleología con lo que se logre proyectar investigación que aumente el conocimiento científico sobre esas cavernas y de sustento a mejor información para el sector turismo.

Por medio de observaciones y pruebas cualitativas se concluye que las cuevas han sido formadas en un complicado sistema hidrogeológico que afecta formaciones de rocas calizas, en donde el principal mineral formador de la morfología kárstica observada es calcita. El artículo es propositivo en la medida de que ahonda en las recomendaciones sobre las investigaciones científicas que puedan realizarse para profundizar más en el conocimiento de dichas cavernas.

Palabras clave: Caverna, karsismo, caliza, calcita, espeleotemas, espeleología.

\section{ABSTRACT}

The present work is the result of an interuniversity project between academics from Universidad Nacional Autónoma de Honduras (UNAH), Universidad de Costa Rica and Grupo Espeleológico Anthros professionals.

An exploratory visit was performed within Taulabé Caves for scientific evidence that could explain the origin and the cave chemical composition in addition to improve

*Universidad Nacional Autónoma de Honduras. Facultad de Química y Farmacia.

urbizovivascesarmauricio@yahoo.es

** patrickduran@gmail.com; marinoperaldo@ucr.ac.cr; rgeoramirez@gmail.com 
academic ties between the involved institutions and to provide scientific information to tourism.

Through observation and qualitative evidence is concluded that the caves have a karst formation and are mainly composed of calcite. Scientific search is recommended to improve the cave knowledge.

Keywords: Cave, karst, limestone, calcite, espeleothems, espeleology 


\section{INTRODUCCIÓN}

El propósito de esta investigación es recabar información básica preliminar sobre el proceso de formación y características químicas de las Cuevas de Taulabé. Mancomunando esfuerzo, conocimiento y experiencia entre diversos campos del saber, se desea determinar el potencial científico de la caverna para desarrollar futuras investigaciones en conjunto que coadyuven al desarrollo del área, usando el sistema kárstico como una oportunidad de desarrollo social y económico de los pobladores de las cercanías, con lo que se propicia la conservación de tal monumento natural.

Las Cuevas de Taulabé representan un atractivo turístico importante, son fuente de trabajo y divisas para la región. Estudios científicos previos de las cavernas son escasos, dispersos, y algunos de difícil acceso. Los datos obtenidos serán de gran utilidad para dependencias del estado e instituciones como el Ministerio del Turismo, Instituto Hondureño de Antropología e Historia, Instituto Hondureño de Ciencias de la Tierra y otras, además de una mejor ilustración a quienes visiten las cuevas.

\section{MARCO TEÓRICO}

Flujos naturales de agua, hielo o lava pueden labrar algunos tipos de terreno provocando la formación de cavidades llamadas "Cuevas o Cavernas". El ejemplo más común de formación de cuevas son las producidas por corrientes de agua ligeramente ácidas que disuelven la roca caliza, un tipo de roca compuesta principalmente de calcita, un mineral de carbonato de calcio $\left(\mathrm{CaCO}_{3}\right)$. Este fenómeno se le conoce como "Karstificación". El dióxido de carbono $\left(\mathrm{CO}_{2}\right)$ gaseoso disuelto en el agua es el responsable de conferirle a ésta un $\mathrm{pH}$ ácido. La calcita es susceptible a las reacciones con ácido. Las reacciones químicas involucradas se describen a continuación:

El proceso químico que se lleva a cabo es el siguiente:

\section{Reacciones Químicas de la Karstificación}

I Fase: Acidulación del agua

- El dióxido de carbono se disuelve en el agua y produce ácido carbónico $\left(\mathrm{H}_{2} \mathrm{CO}_{3}\right)$

$$
\mathrm{CO}_{2}+\mathrm{H}_{2} \mathrm{O} \leftrightarrow \mathrm{H}_{2} \mathrm{CO}_{3}
$$


- El ácido carbónico en agua forman los iones hidronio $\left(\mathrm{H}_{3} \mathrm{O}^{+}\right)$, responsable de la acidez, e hidrogenocarbonato $\left(\mathrm{HCO}_{3}{ }^{-}\right)$

$$
\mathrm{H}_{2} \mathrm{CO}_{3}+\mathrm{H}_{2} \mathrm{O} \rightarrow \mathrm{H}_{3} \mathrm{O}^{+}+\mathrm{HCO}_{3}^{-}
$$

II Fase: Disolución de la roca caliza

- El ión hidronio ataca al carbonato de calcio con la consecuente formación de sus respectivos productos:

$$
\mathrm{H}_{3} \mathrm{O}^{+}+\mathrm{CaCO}_{3} \leftrightarrow \mathrm{Ca}^{2+}+\mathrm{HCO}_{3}^{-}+\mathrm{H}_{2} \mathrm{O}
$$

Un fenómeno característico de las cuevas ocurre cuando el agua de la roca disuelta se evapora y libera dióxido de carbono gaseoso al ambiente, provocando la precipitación de la calcita (carbonato de calcio).

La reacción química correspondiente se detalla a continuación:

$$
\mathrm{Ca}^{2+}+2\left(\mathrm{HCO}_{3}^{-}\right)=\mathrm{CaCO}_{3}+\mathrm{H}_{2} \mathrm{O}+\mathrm{CO}_{2}
$$

La ciencia encargada del estudio de estas formaciones geológicas se conoce como "Espeleología". A las personas que ejercen esta rama del conocimiento se les conoce como espeleólogos. Para practicar la espeleología se necesita, además del conocimiento científico sobre cuevas, una condición física apropiada y un adecuado entrenamiento en técnicas de alpinismo. Es indispensable adquirir el equipo de seguridad profesional (casco, lámparas, botas, cuerdas, arnés, mosquetones, etc.) para un buen desarrollo de la actividad. Algunas personas practican la espeleología más como un deporte de aventura, por los riesgos que implica, que como una ciencia.

Llama la atención los reflejos de los haces de luz que impactan los espeleotemas de las cavernas, proyectan diferentes colores y tonos, lo que haría pensar que existen diversos minerales en la composición de los cristales que conforman dichos espeleotemas. Sin embargo, al tratarse únicamente de calcita, estos diferentes colores y tonos se explican en función de contaminantes existentes en los cristales, que pueden ser arcillas, limos, óxidos de hierro, entre otros, aparte de que existe una disposición sumamente cuidadosa de luces de diferentes colores que aumentan dichas sensaciones lumínicas. 
Las "Estalactitas" son depósitos de calcita acumulados. Por gravedad el agua fluye hacia abajo, al evaporarse permite la formación de estructuras cónicas descendentes.

Las "Estalagmitas" se forman por las gotas de roca disuelta que caen de la estalactita al suelo, al evaporarse el agua forma estructuras cónicas ascendentes. Otros tipos de cuevas:

- Cuevas Marinas son producidas por el mar que desgasta las rocas costeras.

- Cuevas de Hielo son las formadas a partir del derretimiento del hielo en glaciares.

- Cuevas de Lava son las cavidades resultantes del enfriamiento de la parte externa de una colada, mientras que el centro de ella permanece líquida y puede fluir dejando luego una estructura similar a un tubo.

Además de la importancia geológica de las cavernas, también de manera general se puede decir que los sistemas kársticos en varias partes del mundo, tal como en el mundo maya, han sido sitios de estudios antropológicos debido al carácter místico que dichos sistemas, junto con sus espeleotemas, han despertado en las diferentes culturas. En el caso de América Central, en la región cultural mesoamericana, la cultura maya integró los sistemas kársticos de la península de Yucatán dentro de su particular cosmovisión, tal como se indica en un reportaje del sitio digital Artículo 7 (http://a7.com.mx/vida/horizonte-academico/1052-avanza-el-registro-decuevas-y-cavernas-mayas-en-la-region-puuc.html).

En el reportaje referido, se indica que han sido descubiertos restos culturales tales como "...muros con mampostería, accesos subterráneos con dinteles y jambas, petrograbados, materiales como metates, vasijas y platos, y en ocasiones hasta pintura mural sobre las paredes de las cuevas...".

Con relación a las cuevas de Taulabé, no se han localizado vestigios culturales debido, entre otros aspectos, a que la entrada principal conocida actualmente para dichas cavernas no existía como tal antes de la construcción de la carretera a Yojoa, por lo que estuvo hasta el presente vedada en ese sector a los grupos humanos del área cultural prehispánico de Yojoa. No significa esto que no puedan localizarse en otras áreas cercanas, entradas naturales del sistema kárstico donde sí se puedan localizar restos culturales, para lo cual la investigación en dicho sistema debe ser interdisciplinario, en donde se involucren antropólogos y arqueólogos para su estudio sistemático. 


\section{CUEVAS DE TAULABÉ}

Las Cuevas de Taulabé se encuentran a $140 \mathrm{~km}$ de Tegucigalpa, en el departamento de Comayagua, entre el Lago de Yojoa y Siguatepeque. Son consideradas monumento natural y fueron descubiertas accidentalmente en el año 1969 por una compañía israelí que trabajaba en la construcción de la carretera entre San Pedro Sula y Tegucigalpa. En marzo de 1979 Tom Miller y otros dos espeleólogos realizaron el primer plano de la caverna, tipografiando $921 \mathrm{~m}$ sin encontrar el final ya que esta se ramifica y profundiza conforme se avanza. La sala más alta registrada hasta el momento tiene aproximadamente $20 \mathrm{~m}$ de altura en roca caliza, la temperatura varía entre $28-30^{\circ}$ y está situada a $620 \mathrm{msnm}$.

\section{MARCO GEOLÓGICO}

Las Cuevas de Taulabé se encuentran constituidas geológicamente por rocas calizas pertenecientes al Grupo Yojoa. Este grupo está conformado por calizas marinas someras descritas como micritas limosas gris claro a café grisáceo, bien estratificadas, en capas de 10 a $70 \mathrm{~cm}$, muy fosilíferas con abundantes bancos arrecifales de rudistas, que corresponden con bivalbos extintos, a través de la sección y estratos masivos con orbitilina hacia la base (Curran, 1981). Se debe entender que dichas cavernas se enmarcan dentro de un sistema kárstico mayor que debe ser estudiado al detalle a partir de levantamientos geológicos de detalle. Además, la dinámica kárstica del área se debe interpretar al estudiar a profundidad las características hidrogeológicas del sistema.

El Grupo Yojoa posee una edad del Cretácico Inferior al Cenomaniano, y se encuentra sobreyaciendo a la Formación Agua Fría (Mills et al., 1967; Scott y Finch, 1999). Dentro de este grupo, se reconocen dos unidades de plataforma carbonatada: La Formación Atima Superior e Inferior, localmente separadas por las lutitas Mochito (Scott y Finch, 1999).

\section{SISMICIDAD HISTÓRICA DE COMAYAGUA}

Un aspecto a tomar en consideración para incluir en la gestión que se pueda realizar en las cavernas de Taulabé, es la sismicidad cercana, que ha ocurrido en el graben de Comayagua. Uno de los primeros temblores conocidos y que está bien documentado, es el ocurrido el 14 de octubre de 1774. Peraldo y Montero (1999) estudian este sismo y refieren que afectó significativamente los edificios públicos y privados de Comayagua. Se ha referido que hundió el techo de la cárcel y cuartel de Dragones. Afectó severamente la nave central de la catedral. Los conventos de San 
Francisco y La Merced también resultaron con daños de importancia. En Lejamaní afectó las torres del templo, produjo deslizamientos en el Cerro Gorra de Cuero, donde existían unas minas, y produjo otros deslizamientos importantes cerca de Lejamaní y en el área conocida durante el periodo colonial como Las Piedras. En Ajuterique parece que provocó menos daños que en las anteriores localidades referidas. Se le asigna a estos sismos epicentro cercano a Comayagua y una $M_{s} 5,4$ a 6,0. La intensidad calculada es de VII a VIII en Comayagua, VII en Lejamaní y en Las Piedras y VI en Ajuterique. Una referencia a retumbos y una continuación de los sismos réplica sugieren la cercanía del epicentro a los lugares referidos.

Otro significativo sismo que estudian Peraldo y Montero (1999) es el ocurrido el 20 de junio de 1809, que volvió a afectar significativamente la ciudad de Comayagua. En esta ciudad aparte de dañar los edificios que se reportaron para 1774, también dañó considerablemente el Hospital. La intensidad calculada para este sismo por los citados autores es de $\mathrm{VII}$ a VII+ y una magnitud $\mathrm{M}_{\mathrm{s}} 5,7$. Existen más referencias a sismos en 1610 que no están bien documentados, sin embargo, esto indica que el factor sísmico es de vital importancia de ser tomado en consideración para estimar niveles de amenaza y prever el desprendimiento de sectores del techo de la caverna, tal como se observa en la entrada de la caverna situada contiguo a la abierta al público. Un bloque decamétrico se observa caído en la entrada de la caverna, esto significa que existen fracturas o planos de buzamiento en la formación carbonatada que favorecen este tipo de desprendimientos.

No obstante, con un proceso de investigación donde pueda localizarse nueva información sobre los sismos de 1774 y de 1809, podrían variar las estimaciones de los parámetros asignados por Peraldo y Montero (1999). Se hace necesario, a su vez, estudiar a profundidad la neotectónica del área de Comayagua y alrededores para obtener mejor estimación de la amenaza sísmica sobre las cuevas de Taulabé.

\section{DESCRIPCIÓN GENERAL DEL TRAMO DE LA CAVERNA EXPLORADA}

El 16 de julio de 2011 se realizó una visita de reconocimiento a estas cavernas bajo la iniciativa de César Urbizo Vivas, profesor de química de la UNAH (Universidad Nacional Autónoma de Honduras); Patrick Durán Leiva y Giovanni Peraldo, profesores de la Escuela Centroamericana de Geología de la Universidad de Costa Rica (UCR) y Ronald Ramírez Salazar, estudiante de último año de la carrera en Geología de la UCR y miembro del Grupo Espeleológico Anthros de Costa Rica (ver imagen 1). 


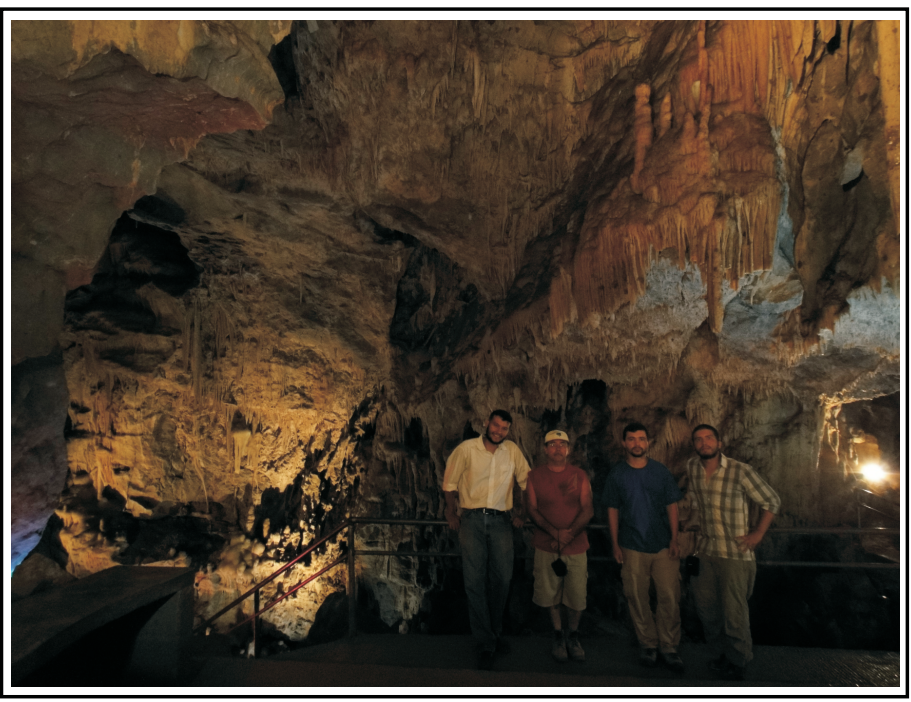

Imagen 1. De izquierda a derecha: César Urbizo Vivas, Giovanni Peraldo Huertas, Patrick Durán Leiva y Ronald Ramírez Salazar. Se aprecian las estalactitas y la forma irregular de la superficie.

La caverna está destinada al turismo, la entrada está acondicionada para todo el público. Los accesos actuales no son los accesos naturales de la caverna, fueron abiertos cuando construyeron la carretera (ver imagen 2).

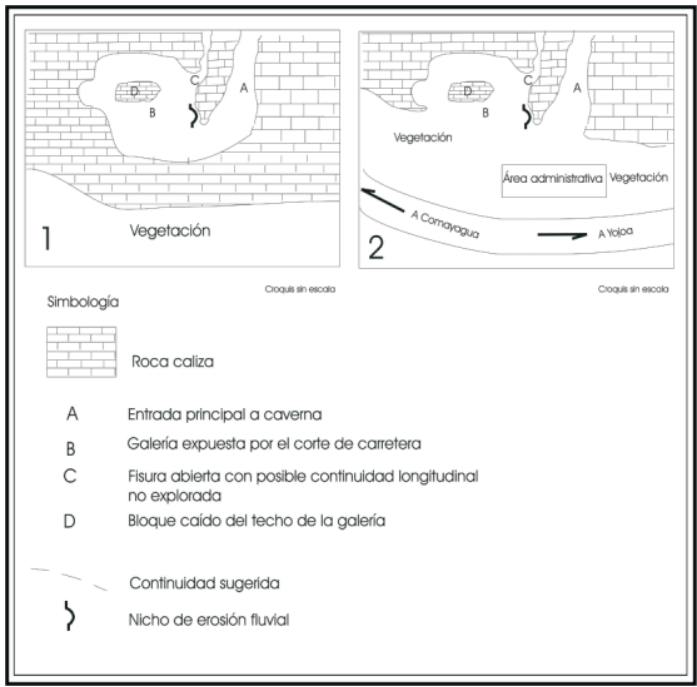

Imagen 2. Croquis de la parte externa de la caverna de Taulabé. A) Posible apariencia de la caverna antes de abrir la trocha para la carretera a Yojoa. B) Apariencia en la actualidad. Se sugiere que las galerías $\mathrm{A}$ (galería abierta al público) y la B (galería angosta a la par de la A) corresponden a una misma galería, solamente que durante la construcción de la carretera fue destruida la pared que la ocultaba. Es importante realizar una expedición espeleológica por la galería B. 
La imagen 2 muestra las dos galerías descubiertas al abrir la trocha para la carretera Comayagua - Yojoa. En la apertura de la trocha descubrieron la caverna de Taulabé. Sugerimos que la galería B corresponde con una sección de la caverna de Taulabé, no es una caverna independiente, responde a una curva de la caverna. Esto se confirma por el hallazgo de lo que parece ser un nicho de erosión por dilución de los carbonatos de la caliza, debido a corrientes de agua. Esta evidencia sugiere un flujo continuo de agua que en el área formó una curva durante la formación de la caverna. Ese rasgo fluvial insinúa una comunicación expedita entre ambas galerías.

El recorrido en el área acondicionada para todo público consta de $300 \mathrm{~m}$ acompañados de un guía turístico, cuenta con accesos de cemento y barandillas de seguridad (ver imagen 3), además de iluminación artificial que facilita el desplazamiento dentro de la caverna y brinda un importante atractivo a los visitantes al iluminar con diferentes colores los diversos espeleotemas de la caverna.

Está la opción de $300 \mathrm{~m}$ adicionales propios para la aventura, los que no cuentan con los servicios de luz ni barandas de seguridad.

Paralelo al recorrido turístico se encuentra otra vía de la caverna, una continuación del trayecto natural, el cual fue perturbado durante la elaboración de la carretera dejando lo que se podría interpretar como otra entrada. Este acceso no ha sido explorado por guías, turistas, ni espeleólogos incluyendo a Tom Miller

El potencial de la caverna es muy alto ya que los sectores observados durante el recorrido turístico presentan desarrollo tanto vertical como horizontal de los pasajes de la caverna, además se observan evidencias de varios eventos de erosión, tales como canales que se pueden observar en el techo de la galería principal, esto se puede deber a un paleoflujo subterráneo

Algunas especies de seres biológicos son capaces de adaptarse y vivir bajo condiciones extremas, al amparo de las fuentes de luz artificial.

La humedad y flujo de agua evidencia un proceso activo de formación de estalactitas. Algunas de ellas se presentan de forma alineada, lo cual sustenta la existencia de patrones importantes de fisuras en la roca, que permiten la percolación de agua desde niveles acuíferos superiores, permitiendo a su vez la destrucción progresiva de la caliza por disolución del carbonato de calcio.

Un experimento cualitativo de campo es la identificación de calcita (carbonato de calcio), aprovechando la sensibilidad del mineral de reaccionar con ácidos. Se toma 
una muestra y se le agrega unas gotas de solución diluida de ácido clorhídrico $(\mathrm{HCl}$ al 10\%). El carbonato de calcio en presencia de ácidos desprende dióxido de carbono que se detecta como efervescencia.

La reacción química es la siguiente:

$$
\mathrm{CaCO}_{3}+2 \mathrm{H}^{+} \rightarrow \mathrm{Ca}^{2+}+\mathrm{H}_{2} \mathrm{O}+\mathrm{CO}_{2} \text { (gas) }
$$

En el trayecto al interior de la caverna se puede observar algunas estructuras de curiosas formas que sugestionan los sentidos.

\section{CONCLUSIONES}

- Las Cuevas de Taulabé se enmarcan en un sistema kárstico que debe ser mejor estudiado, del cual se puede extraer valiosa información desde el punto de vista geológico, hidrogeológico, mineralógico y biológico para reconstruir la historia natural del área.

- Las Cuevas de Taulabé están rodeadas de leyendas y misticismo, el cual es muy atractivo para la investigación científica al intentar revelar los "misterios" de este lugar y poder aportar al conocimiento geocientífico, biológico, espeleológico y cultural de Honduras.

- La cartografía completa de la caverna es la prioridad para poder realizar las investigaciones y poder ubicar espacialmente los resultados.

- Las Cuevas de Taulabé poseen un alto potencial para ser reconocido como un gran sistema de cavernas. Entre las leyendas se dice que este sistema podría comunicarse con varios lugares como: Santa Bárbara, Lago de Yojoa, Copán y LaPaz.

- El sistema kárstico del área es una oportunidad de desarrollo socioeconómico de las poblaciones del área, por ende las personas de esas comunidades deben proteger dicho sistema de la destrucción ambiental antrópica.

\section{RECOMENDACIONES}

- Se recomienda una exploración de al menos una semana completa para realizar la cartografía del sistema kárstico dentro del cual se enmarca la caverna de Taulabé, mediante un levantar la topografía al detalle de la(s) caverna(s) y de geofísica.

- El proceso de levantamiento geológico, reconocimiento de flora y fauna, análisis químico de las aguas subterráneas y análisis mineralógico sería paralelo al avance en el conocimiento cartográfico del sistema kárstico de Taulabé. 
- Realizar una cooperación conjunta entre la UNAH, la UCR y grupos especialistas en espeleología, tal como en Costa Rica el grupo Anthros, para llevar a cabo un estudio de mayor profundidad sobre geología, sismicidad reciente y aceleraciones in situ, para identificar las amenazas para los visitantes de la caverna.

- Estimular una coparticipación entre los diferentes actores sociales de las áreas cercanas y el gobierno, a fin de que el sistema kárstico pueda ser interpretado como una fuente de atracción de riqueza para las comunidades cercanas, en la medida que dichas comunidades se involucren en procesos locales de protección de ese patrimonio geológico hondureño.

\section{AGRADECIMIENTOS}

En primer lugar a Dios por la oportunidad de realizar este proyecto en conjunto y concluido de forma satisfactoria. Agradecemos al equipo de guías turistas de las Cuevas de Taulabé por la gentil colaboración y el apoyo brindado, especialmente a Nahún Portillo Ramírez por su amabilidad e invaluable información proporcionada. También gracias a todas las personas que ocupan un lugar especial en nuestra vida y que de manera indirecta contribuyeron en la investigación con su confianza y motivación: Dra. Marilyn Guevara Peña, César Darío Urbizo, Mauricio Javier Urbizo y Lic. Abril Urbizo Vivas.

Se desea agradecer al PNUD en la persona del Dr. Ginés Suárez y a la Alcaldía de Tegucigalpa porque sin su ayuda el encuentro entre académicos hondureños y costarricenses no hubiera sido posible como tampoco la bella aventura de viajar al corazón de Taulabé. Al M.Sc. Rolando Mora Chinchilla como Director de la Escuela Centroamericana de Geología por depositar toda su confianza en el grupo de profesores que estuvimos en Honduras dirigiendo el curso (G-5216) Campaña Geológica del año 2011. Este artículo es un importante valor agregado a la labor que se realizó en la hermana República de Honduras por parte de la Universidad de Costa Rica. Gracias a la gente linda de Honduras que nos recibió con los brazos abiertos.

\section{BIBLIOGRAFÍA}

Mills, R. A.; Hugh, K. E.; Feray, D. E.; and Swolfs, H. C., "Mesozoic Stratigraphy of Honduras". American Association of Petroleum Geologists Bulletin, v. 51, 1967, p. 1711-1786.

Curran, D., "Mapa Geológico de Honduras". Hoja de Taulabé: escala 1:50,000. Instituto Geográfico Nacional, Tegucigalpa, Honduras, 1981. 
Scott, R. y Finch, R., "Cretaceous Carbonate Biostratigraphy and Environments in Honduras". En: Mann, P. (ed.): Caribbean Basins. Sedimentary Basins of the World, v. 4, 1999, p. 151-166.

Peraldo, G. y Montero, W. "Sismología Histórica de América Central". Instituto Panamericano de Geografía e Historia”. México. 169-172 y 196-197.

http://www.showcaves.com/english/misc/caves/Taulabe.html http://www.anthros.org/

http://a7.com.mx/vida/horizonte-academico/1052-avanza-el-registro-decuevas-y-cavernas-mayas-en-la-region-puuc.html

Consultas efectuadas el día 3 de mayo de 2012 a las 14:00 horas. 